and curve $b$ to $c$ took place. It was possible to reproduce curve $c$ with the circular and annular orifices only, and the transition from curve $b$ to $c$ occurred at a lower rate of swirl with the circular orifice. No qualitative change in the flow pattern could be seen between a point $8 \mathrm{in}$. above the outlet and the tank bottom, which was 6 in. below the inlet.

So far no satisfactory explanation of this phenomenon has been found. It seems to be related to the reverse flow at the centre of a Ranque-Hilsch vortex tube, although no thermal effects are present in this case.

These experiments were made in the Hydraulics Laboratory of the City and Guilds College, London, by permission of Prof. C. M. White.

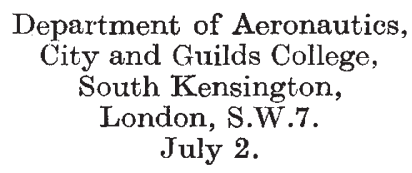

\section{Importance of Ion Exchange with the Solvent in Kinetic Studies}

Is kinetic examination of nucleophilic substitution and elimination reactions with various attacking ions $Y^{\prime}$ in alcohol solution, Bunnett and Zahler ${ }^{1}$ directed attention to complications likely to arise from the replacement of $Y^{\prime}$ by $\mathrm{OEt}^{\prime}$ consequent upon the equilibrium :

(I) $\quad Y^{\prime}+$ HOEt $\rightleftharpoons \mathrm{HY}+\mathrm{OEt}^{\prime}$.

In extension of our earlier work ${ }^{2}$ on the effect of various nucleophilic reagents on the simultaneous substitution (II) and carbonyl elimination (III) reactions of organic esters of nitric acid, we have now obtained definite evidence of the great importance of such ion replacement reactions. We record below the separate values of the second-order velocity coefficients (in gm.mol.-1 lit.sec.-1) for the reactions :

(II) $Y^{\prime}+\mathrm{PhCH}_{2} \cdot \mathrm{O} \cdot \mathrm{NO}_{2} \rightarrow \mathrm{PhCH}_{2} Y+\mathrm{NO}_{3}^{\prime}$

and (III) $Y^{\prime}+\mathrm{PhCH}_{2}$. O. $\mathrm{NO}_{2} \rightarrow \mathrm{PhCH}: \mathrm{O}+$

$$
\mathrm{HY}+\mathrm{NO}_{2}^{\prime}
$$

for attack by initially added $Y^{\prime}=\mathrm{OEt}^{\prime}, \mathrm{OH}^{\prime}$ and $\mathrm{OPh}^{\prime}$ on benzyl nitrate in 100 per cent ethanol solution at $60 \cdot 2^{\circ}$.

The nearly equal values (Nos. 1 and 2) of the velocity coefficients for $\mathrm{OEt}^{\prime}$ and $\mathrm{OH}^{\prime}$ suggest that actual attack is essentially by OEt' in both experiments, in harmony with the independent findings of Caldin and Long.

With OPh' (No. 3), the value of $k E_{\mathrm{CO}} 2$ exhibits a marked downward drift as the reaction proceeds. Such a drift should occur if the formation of free phenol in reaction (III) causes a gradual displacement of the equilibrium (I, $\left.Y^{\prime}=\mathrm{OPh}^{\prime}\right)$ towards the left as the reaction proceeds. Initial addition of free

\begin{tabular}{|c|c|c|c|c|c|c|}
\hline \multirow{2}{*}{$\begin{array}{l}\text { Exp. } \\
\text { No. }\end{array}$} & \multirow{2}{*}{$Y^{\prime}$} & \multirow{2}{*}{$\begin{array}{l}\text { Added } \\
\text { H } Y\end{array}$} & \multicolumn{2}{|c|}{ Initial concentrations } & \multicolumn{2}{|c|}{$\underbrace{10^{5} k_{2}}$} \\
\hline & & & {$\left[\mathrm{PhCH}_{2} \mathrm{NO}_{3}\right]$} & {$\left[Y^{\prime}\right]$} & $S_{N 2}$ & $E \mathrm{CO} 2$ \\
\hline 1 & OEt' & 一 & 0.0301 & $0 \cdot 0366$ & $96 \cdot 7$ & 1,350 \\
\hline 2 & $O \mathrm{H}^{\prime}$ & - & 0.0265 & 0.0406 & 99 & 1,440 \\
\hline 3 & $O P h^{\prime}$ & $0 \cdot 006 M$ & 0.0498 & 0.0946 & $74 \cdot 2$ & $48.6 \rightarrow 36.9 *$ \\
\hline 4 & $O P h^{\prime}$ & $0.236 \mathrm{M}$ & 0.0524 & $0 \cdot 101.0$ & $60 \cdot 5$ & $6.3=0.094$ \\
\hline 5 & oph' & $0 \cdot 322 M$ & 0.0478 & 0.0897 & $61 \cdot 4$ & $5 \cdot 3 \pm 0.06 t$ \\
\hline
\end{tabular}

* Coefficient drifts throughout run. † Coefficient constant thoughout run. phenol to the reaction mixture, in concentrations sufficient to swamp the small amount produced in reaction (III), should, by a mass law effect, cause more-or-less complete suppression of OEt'. The experimental value of $k E_{\mathrm{CO}} 2$ thus obtained should approach the true value for attack by phenoxide ion.

It was found that such additions of free phenol in increasing concentrations $(a)$ cause successive decreases in $k E_{\mathrm{CO}} 2$ towards an asymptotic value, and $(b)$ gradually eliminate the drift in the constant. In the presence of $0.3 M$ phenol (No. 5) $k E_{\mathrm{CO}} 2$ has been reduced to about one-ninth of the original value and remains constant throughout the experiment. The true value of $10^{5} k^{2} E_{\mathrm{CO}} 2$ for $\mathrm{OPh}^{\prime}$ cannot, therefore, be greater than $5 \cdot 3$, and extrapolation suggests that it may be as low as 2-3. Within this range, however, the actual value makes no significant difference in the following deductions.

This value, together with that for ethoxide ion (No. 1), permits calculations of the changing concentration of $\mathrm{OPh}^{\prime}$ and $\mathrm{OEt}^{\prime}$ present throughout experiment No. 3. They reveal that, although $\left[\mathrm{OPh}^{\prime}\right] /\left[\mathrm{OPh}^{\prime}\right]+\left[\mathrm{OEt}^{\prime}\right]=0.97 \rightarrow 0.98$, because of the much greater nucleophilic power of $\mathrm{OEt}^{\prime}$, approximately 90 per cent of the total carbonyl elimination is effected by ethoxide ion. Even in experiment No. 5 it is still approximately 10 per cent. Incidentally, it will be noticed that the replacement of OEt' by $\mathrm{OPh}^{\prime}$ has a much smaller effect on ${ }_{k} S_{N} 2$ than on $k E_{\mathrm{CO}} 2$, in agreement with the well-known fact that, with decreasing basic strength, the nucleophilic power of the reagent towards hydrogen diminishes much more rapidly than it does towards carbon.

Our data also permit an approximate assessment of the value of the equilibrium constant $K=$ [OEt'] [PhOH] $] /\left[\mathrm{OPh}^{\prime}\right][\mathrm{EtOH}]$ as $\sim 10^{-5}$.

The important implications of these conclusions on the validity of previously published results for attack of various nucleophilic ions in proton-donating solvents are obvious. Our investigations are being extended and will be published in full elsewhere when they are complete.

Department of Organic Chemistry,

$$
\text { J. W. BAKER }
$$

A. J. Neale

University, Leeds 2. June 17.

${ }^{3}$ Bunnett, J. F., and Zahler, R. E., Chem. Rev., 49, 399 (1951) ; cf. Cox, H. E., J. Chem. Soc., 113, 666 (1918).

${ }^{2}$ Baker, J. W., and Easty, (Mrs.) D. M., Nature, 168, 156 (1950); J. Chem. Soc., 1193, 1208 (1952).

${ }^{3}$ Caldin, E. F., and Long, $G$. (following communication).

\section{Equilibrium between Ethoxide and Hydroxide Ions in Ethanol}

THERE is some evidence ${ }^{\mathbf{1 , 2}}$ that, in solutions made by dissolving sodium hydroxide in ethanol, much of the hydroxyl ion is replaced by ethoxide, the equilibrium $\mathrm{OH}^{-}+\mathrm{EtOH} \rightleftharpoons \mathrm{OEt}^{-}+\mathrm{H}_{2} \mathrm{O}$ lying well to the right. We have confirmed this by measuring the equilibrium constant $\dot{K}=\left[\mathrm{OEt}^{-}\right]\left[\mathrm{H}_{2} \mathrm{O}\right] /\left[\mathrm{OH}^{-}\right][\mathrm{EtOH}]$ at $25^{\circ} \mathrm{C}$., using solutions made by dissolving sodium or sodium hydroxide in ethanolwater mixtures containing 10-50 per cent water by weight. The method adopted makes use of the fact that trinitrotoluene ionizes to give an intensely coloured ion in the presence of ethoxide ${ }^{3}$. 